Spallone, A., et al. Measurements Of The Temperature Coefficient Of Electric Resistivity Of Hydrogen Overloaded Pd. in The 12th International Conference on Condensed Matter Nuclear Science. 2005. Yokohama, Japan.

\title{
MEASUREMENTS OF THE TEMPERATURE COEFFICIENT OF ELECTRIC RESISTIVITY OF HYDROGEN OVERLOADED PD*
}

\author{
Antonio SPALLONE, Francesco CELANI \\ INFN-LNF, Via Enrico Fermi \\ 00044 Frascati (Rome), Italy \\ Paolo MARINI, Vittorio Di STEFANO \\ EURESYS, Via Lero 30 \\ 00129 Rome, Italy
}

\begin{abstract}
As reported in previous papers, we performed many electrolytic loading tests using thin Pd wires, achieving loading ratios of $\mathrm{H} / \mathrm{Pd} \geq 0.95$ (H/Pd over-loading). In particular, we defined a reproducible "loading protocol" suitable for achieving such an over-loading level, based on the use of very diluted acid electrolytic solutions (with additions of tenths of micro-moles of $\mathrm{Ca}$ or $\mathrm{Sr}$ or $\mathrm{Li}$ cations and some hundred nano-moles of $\mathrm{Hg}$ ions) and operating with electrolytic current cycles from a few $\mathrm{mA}$ up to one hundred $\mathrm{mA}$.

By observing the day/night cyclic fluctuations of electrical resistance, as a function of the corresponding temperature variations, of stable, long term, $\mathrm{H} / \mathrm{Pd}$ loadings we were able to calculate the temperature coefficient of resistivity $\left(\mathrm{K}_{\theta}\right)$ of the $\mathrm{Pd}-\mathrm{H}$ system at very high $\mathrm{H} / \mathrm{Pd}$ loadings.
\end{abstract}

Many years ago (on 1998) we reported an unexpected value showing that the $\mathrm{K}_{\theta}$ parameter values increase when $\mathrm{H} / \mathrm{Pd}$ exceeds 0.75 (i.e. after that R/Ro goes beyond the 1.8 peak value, i.e. to the right side of the $\mathrm{R} / \mathrm{Ro} / \mathrm{H} / \mathrm{Pd}$ curve). This fact was confirmed by the ISRStanford Group (McKubre and Tripodi) and Pirelli-Research Group (Gamberale and Garbelli).

In this paper we show several measurements of $\mathrm{K}_{\theta}$ at different overloading values of $\mathrm{H} / \mathrm{Pd}$ up to $\cong 1$ (corresponding at $\mathrm{R} / \mathrm{Ro}=1.12$ ) where $\mathrm{K}_{\theta}=(13 \pm 1) \cdot 10^{-3} \mathrm{~K}^{-1}$, i.e. more than six times higher than the minimum value achieved at the $\mathrm{R} / \mathrm{Ro}=1.8$ peak value.

This result can corroborate the hypothesis that a new Pd-H phase (full $\beta$-phase or the beginning of $\beta+\gamma$ phase) could occur after the $\mathrm{H} / \mathrm{Pd}=0.75$ loading ratio (at the end of $\alpha+\beta$ phase), as claimed by many authors as the necessary condition for excess (anomalous) heat from Pd-D system (at D/Pd $\geq 1$ ).

\section{Historical Background}

\subsection{H/Pd Loading Measurements}

It is common opinion that to observe heat in excess from Pd-D system it is necessary to obtain very high values $(\geq 0.95)$ of $\mathrm{D} / \mathrm{Pd}$ atomic ratio $[\mathbf{1 , 2 , 3 ]}$.

Starting from 1998 we decided to perform systematic tests in order to achieve very high H/Pd loading ratio using very diluted electrolytes and thin (50 or $100 \mu \mathrm{m}$ in diameter) Pd wires as cathodes [4]. Different electrolytic solutions have been tested by adding to the acid solution very low amounts of $\mathbf{C a}, \mathbf{S r}, \mathbf{L i}$ and $\mathbf{H g}$ ions. In this way high $\mathbf{H} / \mathbf{P d}$ loading ratios have been achieved with a satisfactory grade of reproducibility. Several loading procedures have been performed in a wide range of electrolysis current (from a few $\mathrm{mA}$ up to one hundred $\mathrm{mA}$ ) and at different $\mathbf{H g}$ ion concentrations. The obtained results allowed for the definition of a loading protocol capable to insure very high $\mathrm{H} / \mathrm{Pd}$ over-loading. Stable R/Ro $\leq .1 .2$ values (corresponding to $\mathbf{H} / \mathbf{P d}$ ratios $\geq 0.97$ ) can be currently achieved with an extremely low electrolytic power supply (10 V; $5 \mathrm{~mA})[5]$.

This procedure is based on the increase of the cathodic over-voltage (which is known to be the main controlling parameter of the $\mathbf{H}(\mathbf{D})$-Pd loading) obtained by modifying the nature of the cathodic surface (i.e. by inducing the formation of a very thin layer of an alkaline-earth carbonate on its surface).

\subsection{Temperature Coefficient of Resistance trend}

The temperature coefficient of electric resistivity $\left(\mathrm{K}_{\theta}\right)$ of a Pd-H sample is a well known curve in the literature $\left(\mathrm{K}_{\theta}\right.$ is known to decrease from 4.1 to $1.8 \mathrm{mK}^{-1}$ with $\mathrm{H} / \mathrm{Pd}$ increase from 0 to 0.7$)$ [6,7].

During 1998, after the achievement of a very high and stable H/Pd loading $(\cong 0.95)$, we performed a preliminary measurement of $\mathrm{K}_{\theta}$ resulting in a value of $(3.2 \pm 0.2) \cdot 10^{-3} \mathrm{~K}^{-1}$ within a temperature range from $77{ }^{\circ} \mathrm{K}$ up to $373{ }^{\circ} \mathrm{K}$. For the first time in the literature this value showed that at values of $\mathrm{H} / \mathrm{Pd}$ higher than 0.7, $\mathrm{K}_{\theta}$ increases [8,9]. Further, this fact was confirmed by the ISR-Stanford Group [10] and Pirelli-Research Group [11].

\footnotetext{
${ }^{*}$ This work is supported by I.N.F.N.-L.N.F., Frascati, Italy.
} 


\section{Temperature Coefficient Measure}

\subsection{Experimental Setup}

In an electrolytic cell (solution of one liter of $\mathrm{H}_{2} \mathrm{O}+\mathrm{HCl}$ at $10^{-4}$ Moles) the electrodes have been located in the form of 2 parallel wires, of length $25 \mathrm{~cm}, 6,5 \mathrm{~cm}$ apart from each other (the Pd cathode of diameter $50 \mu \mathrm{m}$, the Pt anode of diameter $0.5 \mathrm{~mm}$ ); tenths of micro-moles of $\mathrm{Sr}$ and hundreds of nano-moles of $\mathrm{Hg}$ have been added to the solution. A junction for a pick up of the voltage divided the Pd cathode in 2 equal segments (named: "up" and "down").

\subsection{Experimental Data and Fit}

After applying the procedure to reach H/Pd overloading (OFF/ON current cycles, as shown in Fig. 1), if the loaded sample is found stable, we proceed with the recording of the R/Ro variations following the day/night cyclic temperature variations. The measurement campaign may last for several day/night cycles. To complete the test, $\mathrm{Pd}-\mathrm{H}$ wire is deloaded either by using anodic current or by simply turning the current off for many hours (as reported in Fig. 1) and observing the phase transition at $\mathrm{R} / \mathrm{Ro}=1.7(\mathrm{H} / \mathrm{Pd}=0.6)$.

Generally, as in the latter case, it is possible to check the $R /$ Ro curve tracing back to the peak value $(R / R o=1.78$, $\mathrm{H} / \mathrm{Pd}=0.75$ ) and returning to the initial value of $\mathrm{R} / \mathrm{Ro}=1$. By considering the data in the range where the loading is stable, it is possible to record the dependence of the wire resistance on the wire temperature (homogenous with the cell temperature) and once the linearity of these data is confirmed (Fig. 2), we can make a fit to calculate the $\mathrm{K}_{\theta}$ coefficient according the relation (A.1) reported in the Appendix.

To be consider valid to our purpose, resistance data have to be repetitive after each temperature cycle.

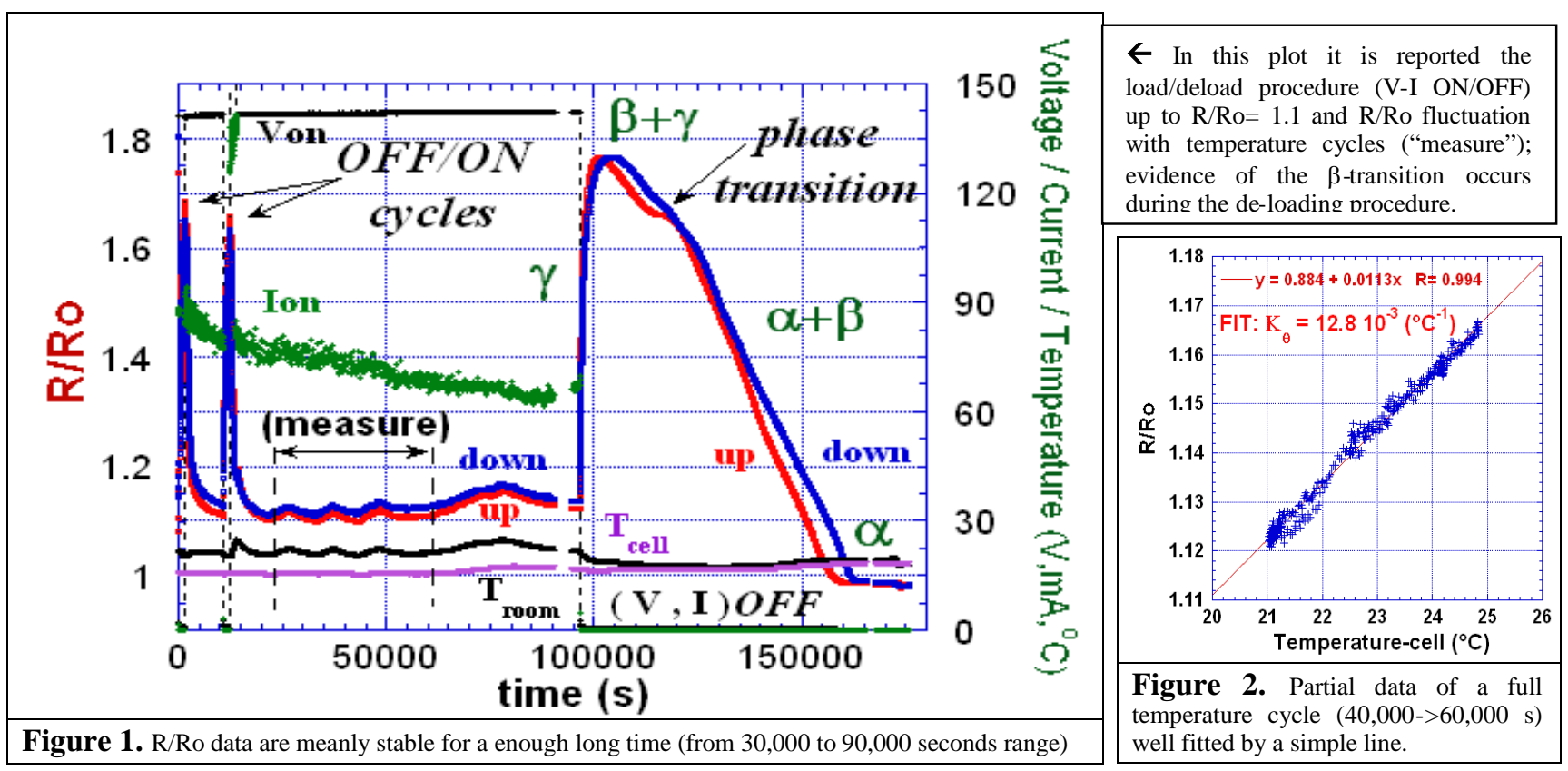

\begin{tabular}{|c|c|c|c|c|c|}
\hline \multirow[b]{2}{*}{ H/Pd } & \multicolumn{2}{|c|}{ (up) } & \multicolumn{2}{|c|}{ (down) } & \multirow[b]{2}{*}{ Note } \\
\hline & $\begin{array}{c}\mathrm{R} / \mathrm{Ro} \\
\pm \mathbf{0 . 1}\end{array}$ & $\begin{array}{c}\mathbf{K}_{\theta} \\
\left(\mathbf{m K}^{1}\right)\end{array}$ & $\begin{array}{r}\mathrm{R} / \mathrm{Ro} \\
\pm 0.1\end{array}$ & $\begin{array}{c}\mathbf{K}_{\theta} \\
\left(\mathbf{m K}^{1}\right)\end{array}$ & \\
\hline 0.00 & 1.0 & $4.1 \pm 0.1$ & 1.0 & $4.1 \pm 0.1$ & (a) \\
\hline 0.85 & 1.75 & $2.8 \pm 0.3$ & 1.75 & $2.6 \pm 0.3$ & (b) \\
\hline $0.88 \div 0.90$ & 1.58 & $7.2 \pm 1$ & 1.61 & $6.7 \pm 0.5$ & (c) \\
\hline 0.91 & 1.52 & $\approx 7 \pm 1.5$ & 1.52 & $\approx 7 \pm 1.5$ & (c) \\
\hline $0.92 \div 0.93$ & 1.44 & $\cong 8.3 \pm 1$ & 1.46 & $\cong 8.4 \pm 1$ & (c) \\
\hline $0.96 \div 0.98$ & 1.19 & $9.9 \pm 0.5$ & 1.24 & $9.6 \pm 0.5$ & (c) \\
\hline $0.97 \div 0.98$ & 1.18 & $10.7 \pm 0.5$ & 1.21 & $10.6 \pm 0.5$ & (c) \\
\hline $0.99 \div 0.995$ & 1.10 & $\cong 13 \pm 1.5$ & 1.13 & $12.8 \pm 0.5$ & (c) \\
\hline
\end{tabular}

Table 1. Values of $\mathrm{K}_{\theta}$ as resulting from several tests performed at stable loading conditions and ratios (ref. Troom at $20^{\circ} \mathrm{C}$ ):

a) The first value is in agreement with the expected value for $\mathrm{K}_{\theta}=4.1$.

b) At the R/Ro peak value the thermal coefficient is a little higher $(30 \%)$ than the one reported in the literature $\left(\mathrm{K}_{\theta} \cong 2.0\right)$.

c) At all values, beyond the R/Ro peak, the thermal coefficient shows rapid increase. 


\subsection{Results}

Out of several hundred tests, only a few tens have shown a loading stability suitable for the calculation of the $\mathrm{K}_{\theta}$ parameter. We report in Table 1 some values corresponding to different values of Pd loading.

In this table, measured values of R/Ro are reported for each of the two wire sectors "up" and "down" (generally showing similar loading values); tests ranged along a period of a year during which different wires, electrolytes and procedures were used.

$\mathrm{H} / \mathrm{Pd}$ values reported in the table are taken from literature data up to R/Ro peak value and after this point we refer to Mc Kubre experimental and theoretical data, as previously reported [12].

In Fig. 3 we report known literature data ("+" points below the R/Ro peak) in addition to the new experimental data ("o" points beyond the R/Ro peak) which show the progressive increase of $\mathbf{K}_{\theta}$ as the H/Pd value increases (overloading); the arrows show the trend of $K_{\theta}$ with increasing loading ratio .

Similarly, in Fig. 4 we report data from the literature and the experiments; they show clearly the rapid progressive increase of $\mathbf{K}_{\theta}$ at $\mathrm{H} / \mathrm{Pd}$ overloading.

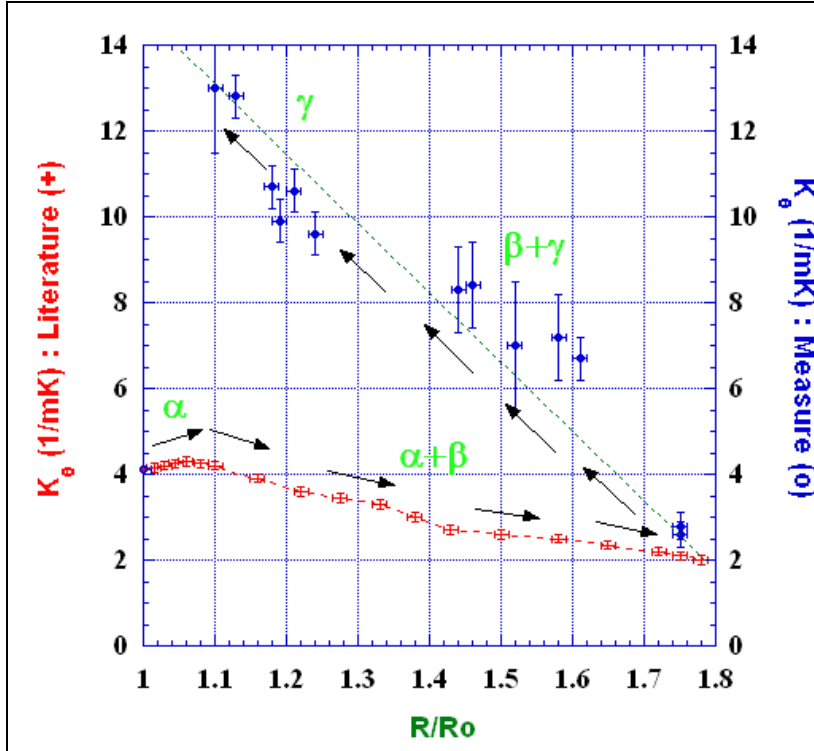

Figure 3. Pd-H temperature coefficient vs normalized resistance of the wire. Experimental data are reported in addition to literature data.

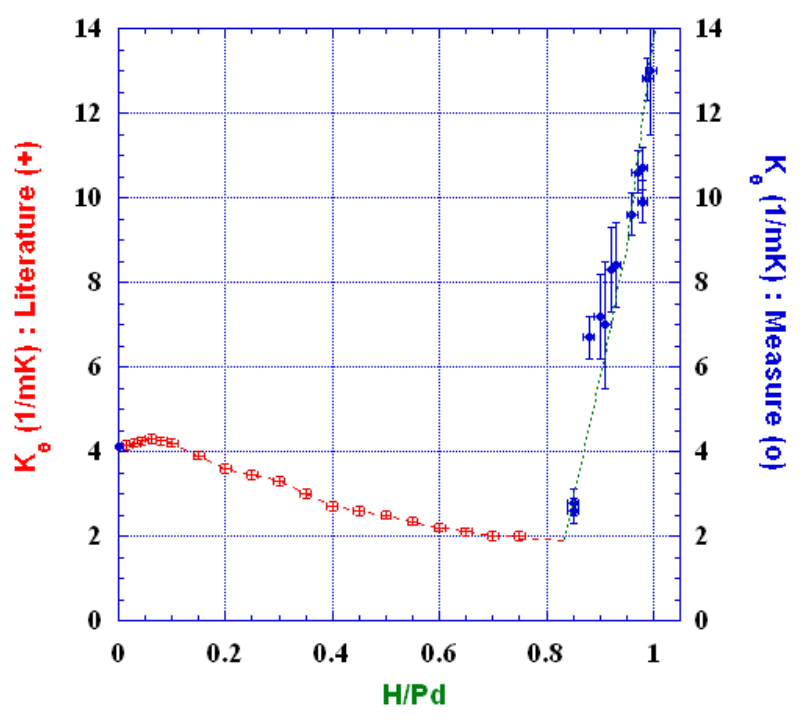

Figure 4. Pd-H temperature coefficient vs normalized $\mathrm{H} / \mathrm{Pd}$ loading. Estimated data, from McKubre tables, are reported in addition to literature data.

\section{Discussion}

\subsection{Phase hypothesis}

From the literature $[\mathbf{2 , 3 , 6 ]}$ is known that at room temperature the system Pd-Hx occurs in different phase conditions $(\alpha-$ phase at $\mathrm{H} / \mathrm{Pd}=0 \rightarrow 0.1$ and $\alpha+\beta$ phase at $\mathrm{H} / \mathrm{Pd}=0.1 \rightarrow 0.6$ ). Because of the change of dimension occurring for $\mathrm{R} / \mathrm{Ro}$ at the peak value of 1.8 when $\mathrm{H} / \mathrm{Pd}$ is ranging from $0.7 \rightarrow 0.8$ [1], it is possible to suppose a phase transition is taking place as it is pointed by an arrow in Fig. 1 at $\mathrm{R} / \mathrm{Ro}=1.7$. At this $\mathrm{R} / \mathrm{Ro}$ peak we hypothesize a new phase, named " $\beta+\gamma$ " phase. If, at high $\mathrm{H} / \mathrm{Pd}$ loading, this R/Ro peak is surpassed, we can assume that a peculiar $\gamma$-phase occurs in correlation with the change of slope of the $\mathrm{K}_{\theta}$ vs temperature curve (Fig.4) so that this new phase is peculiar of H/Pd high loadings.

\subsection{Conclusion}

In conclusion, we stress the following points:

- $\quad$ We are able to achieve $\mathrm{H} / \mathrm{Pd} \cong 1$ staying stable for a long time;

- We could calculate Thermal Coefficient of Resistance at high Pd-H loadings;

- At high loadings as the R/Ro strongly decreases, the thermal coefficient of electrical resistivity strongly increases;

- We can conjecture a new important phase occurring at high loadings. 


\section{Acknowledgments}

We are indebted to Eng. Alfredo Mancini for his precious support. We are grateful to Dr. Daniele Garbelli and Dr Luca Gamberale for their important help and we want to thank Dr. Mike McKubre for his useful suggestions. We like to stress the expertness of Mr Vincenzo Andreassi, our skill technician.

\section{Appendix}

We used the known formula to calculate $\mathrm{K}_{\theta}$ :

$$
\mathbf{R}(\mathbf{x}, \theta)=\mathbf{R o}(\mathbf{x}) \cdot\left(\mathbf{1}+\mathbf{K}_{\theta}(\mathbf{x}) \cdot \theta\right)
$$

Where: $\mathrm{R}$ (in $\Omega$ ) is the electric resistance of the wire (related to a fixed $\mathrm{x}=\mathrm{H} / \mathrm{Pd}$ loading ratio) and depending on the wire temperature $\theta$ (in Celsius Centigrade); Ro is the wire resistance at $0{ }^{\circ} \mathrm{C}$ of temperature. $\mathrm{K}_{\theta}$ is the temperature coefficient of resistance $\left(\right.$ in $\left.{ }^{\circ} \mathrm{C}^{-1}\right)$.

We consider $\mathrm{K}_{\theta}$ constant within a large range of temperature; indeed we have checked this constancy (within $5 \%$ of variation) from $200{ }^{\circ} \mathrm{K}$ up to $373{ }^{\circ} \mathrm{K}$ with a overloaded wire.

\section{References}

1. M.C.H. McKubre et al. Frontiers of Cold Fusion (Proc. ICCF3, 1992, Nagoya, Japan), 5 (1993).

2. B.Baranowski et al., J. Less Common Met., 158, p.347 (1990).

3. B.Baranowski and R. Wisniewski, Phys. Stat. Sol. 35, p.539 (1969).

4. A. Spallone, F. Celani, P. Marini and V. di Stefano, "Experimental studies to achieve H/Pd loading ratio close to 1 in thin wires, using different electrolytic solutions." Proceedings of the $9^{\text {th }}$ International Conference on Cold Fusion, "Condensed Matter Nuclear Science" pg 319-322, Beijing (China), 19-24 May 2002; Edited by Xing Z. Li.

5. A.Spallone et al., "An overview of experimental studies about H/Pd over-loading with thin Pd wires and several different electrolytic solutions." Proceedings of the $11^{\text {th }}$ International Conference on Cold Fusion, Marseilles (France), 01-05 Nov. 2004; Edited by J.P.Biberian. (publishing on Feb. 2006).

6. J.C. Barton, F.A. Lewis and I. Woodward, Trans. Faraday Soc. 59, 1201 (1963).

7. M.C.H. McKubre et al. "Calorimetry and Electrochemistry in the D/Pd System", Proceedings of the First Annual Conference on Cold Fusion, Salt Lake City, Utah, March 28-31, 1990.

8. P.Marini, A.Spallone et al. XXI Secolo Scienza e Tecnologia, Anno X - Num. 1, pg. $34-41$ (1999).

9. F.Celani, A.Spallone et al. "High Hydrogen loading of thin Palladium wires through alkaline-earth carbonates' precipitation on the cathodic surface. Evidence of a new phase in the Pd-H system." INFN: LNF-00/006 (P), 6 Marzo 2000 --- To be published by Physics Letter A .

10. P.Tripodi, M.McKubre et al., Phy.Lett.A, 276, 122-126 (2000).

11. L.Gamberale, D.Garbelli and G.Piana, "Measurement of Heat Capacity of PdHx" Proceedings of the $9^{\text {th }}$ International Conference on Cold Fusion, "Condensed Matter Nuclear Science" pg 105-108, Beijing (China), 19-24 May 2002; Edited by Xing Z. Li.

12. M.C.H. McKubre, (Data reported at the same Conference and Private Communications). 